\title{
Randomised controlled trial of GP-led in-hospital management of homeless people ('Pathway')
}

\author{
Authors: Nigel Hewett, ${ }^{\mathrm{A}}$ Peter Buchman, ${ }^{\mathrm{B}}$ Jeflyn Musariri, ${ }^{\mathrm{C}}$ Christopher Sargeant, ${ }^{\mathrm{D}}$ Penny Johnson, ${ }^{\mathrm{E}}$ \\ Kushala Abeysekera, ${ }^{\mathrm{F}}$ Louise Grant, ${ }^{\mathrm{G}}$ Emily A Oliver, ${ }^{\mathrm{H}}$ Christopher Eleftheriades, ${ }^{\mathrm{I}}$ Barry McCormick, ${ }^{,}$ \\ Aidan Halligan, ${ }^{\mathrm{K}}$ Nadine Marlin, ${ }^{\mathrm{L}}$ Sally Kerry ${ }^{\mathrm{M}}$ and Graham R Foster ${ }^{\mathrm{N}}$
}

\begin{abstract}
Homeless people have complex problems. GP enhanced care (Pathway) has shown benefits. We performed a randomised, parallel arm trial at two large inner city hospitals. Inpatient homeless adults were randomly allocated to either standard care (all management by the hospital-based clinical team) or enhanced care with input from a homeless care team. The hospital data system provided healthcare usage information, and we used questionnaires to assess quality of life. 206 patients were allocated to enhanced care and 204 to usual care. Length of stay (up to 90 days after admission) did not differ between groups (standard care 14.0 days, enhanced care 13.3 days). Average reattendance at the emergency department within a year was 5.8 visits in the standard care group and 4.8 visits with enhanced care, but this decrease was not significant. Quality of life scores after discharge (in 108 patients) improved with enhanced care (EQ-5D-5L score increased by 0.12 [ $95 \% \mathrm{CI} 0.032$ to 0.22 ] compared wtih 0.03 [ -0.1 to $0.15 ; p=0.076]$ with standard care). The proportion of people sleeping on the streets after discharge was $14.6 \%$ in the standard care arm and $3.8 \%$ in the enhanced care arm $(p=0.034)$.
\end{abstract}

Authors: ${ }^{A}$ general practitioner, Medical Director Pathway, University College Hospital Homeless Team, London, UK; ${ }^{\text {B }}$ general practitioner, Health E1 Homeless Medical Centre, London, UK; ' nursing sister, Barts Health NHS Trust, London, UK, and Division of Medical Education, Brighton and Sussex Medical School, Brighton, UK; Deneral practitioner, Brighton and Sussex University Hospital, Brighton, UK; Enursing sister, Brighton and Sussex University Hospital, Brighton, UK; Fresearch fellow, Queen Mary University of London, London, UK; ${ }^{G}$ research fellow, Queen Mary University of London, London, UK; ${ }^{H}$ research fellow, Queen Mary University of London, London, UK; ' research fellow, Centre for Health Service Economics and Organisation, Department of Primary Care, University of Oxford, Oxford, UK; ' professor of economics, Centre for Health Service Economics and Organisation, Department of Primary Care, University of Oxford, Oxford, UK; ${ }^{\mathrm{K}}$ chair, Pathway, RIP, Central Manchester University Hospitals NHS Trust and University

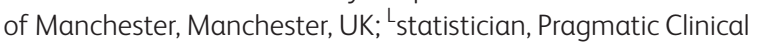
Trials Unit, Queen Mary University of London, London, UK; M reader in medical statistics, Pragmatic Clinical Trials Unit, Queen Mary University of London, London, UK; N professor of hepatology, Queen Mary University of London, London, UK
The quality-of-life cost per quality-adjusted life-year was $£ 26,000$. The Pathway approach doesn't alter length of stay but improves quality of life and reduces street homelessness.

KEYWORDS: Homeless, randomised controlled trial, Pathway, mental health, service provision, addiction

\section{Introduction}

Homelessness is associated with multiple health problems and premature death, and there is growing understanding that long-term homelessness is a health issue. ${ }^{1-3}$ Inadequate care for homeless people leads to increased expenditure, with a minority of homeless people making frequent use of emergency departments. ${ }^{4}$ Annual inpatient costs for a homeless person in England are estimated to be eight times higher than that for the housed population. ${ }^{5}$

Chronic homelessness is characterised by physical and mental ill health and, often, substance misuse. Primary care physicians have the skills to address these issues. ${ }^{6}$ A number of studies have examined housing interventions (such as Housing First) in homeless people with mental health disorders and HIV, ${ }^{7-11}$ but studies in those with medical disorders have not been reported. In 2010, a GP-led and nurse-led intervention involving a hospital 'in reach' team for homeless people at University College Hospital London was developed. This intervention, Pathway, involved GP ward rounds and nurse practitioner patient support with a weekly multi-agency meeting. An audit indicated reduced in-patient stay and an improvement in the quality of care. ${ }^{12}$ We assessed costs and benefits of the Pathway intervention in a randomised controlled trial in two UK centres.

\section{Methods}

We did a randomised, parallel-arm trial in the Royal London Hospital (London) and Royal Sussex County Hospital (Brighton) to test the hypothesis that a complex intervention (ie Pathway) in homeless hospital inpatients leads to a reduction in length of stay. Predefined, secondary endpoints included impact on quality of life, post-discharge accommodation and cost-effectiveness. Homeless patients admitted to hospital were randomised to either standard or enhanced care (the Pathway approach). Hospital ward staff notified the homelessness 
nurse of all admitted patients aged 18 years or older who were homeless (ie who did not have somewhere to stay when they left hospital, including people living with a friend or in a hostel and those who became homeless as inpatients). Exclusion criteria were visitors with an address elsewhere and patients who did not provide consent within 7 days. Readmitted patients who had been randomised to the study were assigned to their original group.

After providing informed consent and baseline assessment, patients were randomly allocated to either standard or enhanced care, using a web-based system. During the study (6 months' recruitment in London), the patient information sheet was modified to allow lifelong follow-up. Randomisation was 1:1, and was stratified by centre. Random-sized blocks (2, 4 or 6) were used to control workload and achieve balance across centres. Neither participants nor health professionals were blinded but primary outcome data from hospital records were cleaned and masked to allocation.

Patients assigned to standard care were visited once by the homelessness health nurse and provided with an information leaflet describing local services. The Pathway approach has been described elsewhere. ${ }^{12}$ Briefly, patients randomised to enhanced care were visited regularly by the homelessness nurse to provide support and establish community links. A GP (PB or CS) performed thrice weekly ward rounds and provided advocacy advice and medical input. A weekly multi-agency meeting was attended by the Pathway team, local council officers, hostel managers, outreach workers, drug and alcohol nurses, homeless centre staff, social and palliative care workers, hospital consultants and therapists. Patients randomised to the enhanced care arm who had challenging discharge needs were discussed and multi-agency care plans devised. Patients randomised to standard care were not discussed. All patients completed a questionnaire detailing current lifestyle and health status before randomisation (admission questionnaire, $\mathrm{S} 1$ ).

Patients were contacted within 6 weeks ( \pm 4 weeks) of discharge by a research fellow and asked to complete a second questionnaire (follow-up questionnaire, S2), either face-toface or by telephone. We planned to contact $25 \%$ of patients at random, but following initial attempts showing that only $25 \%$ of patients responded, we attempted to contact everyone. The study was approved by the UK Ethics Committee, London South East (11/LO/0755).

\section{Study endpoints}

The primary outcome was cumulative duration of hospital stay (time between admission and discharge summed across all admissions within 90 days of initial admission, censored at 90 days). Secondary outcomes included emergency department reattendance 3 months after discharge, readmission 1 and 3 months after discharge, quality of life (measured by EQ-5D-5L $\mathrm{L}^{13}$ and a 10-point sliding scale questionnaire) and accommodation after discharge. An additional, longer-term impact analysis was performed to assess the cumulative length of stay in patients a year from first admission.

\section{Statistical analysis}

Data on patients admitted to the hospitals was collected by the hospital data system and was obtained by regular downloads. The trial team cleaned the data, removed duplication and resolved queries by discussion. Analysis was performed in Stata 11 SE. An audit at University College Hospital ${ }^{12}$ of 250 patients showed that the average length of stay fell from 12.7 to 9.5 days. Our primary outcome was length of stay, which was likely to follow a skew distribution. Using a log transformation, reduction in length of stay of around $18 \%$, with standard deviation in both groups of around 1.0, could be detected with $80 \%$ power at the $5 \%$ significance level if there were 400 participants in each group. In an average year, over 800 homeless people are admitted to the Royal London Hospital and Royal Sussex County Hospital, and we assumed that few patients would not participate. We planned to include all eligible admissions over 12 months from December 2011. Following delays in Brighton (enrolment commenced June 2012) and slow recruitment in London, London recruitment was extended until June 2013. All randomised patients were analysed. A regression model was used to analyse length of stay, adjusting for age, sex and site using bootstrapping to allow for non-normality in the outcome data. The proportion of patients readmitted after discharge was analysed using a logistic regression model adjusting for age, sex and site. Quality of accommodation was assessed as 'living on the street' or 'in accommodation' (including a hostel).

\section{Cost-effectiveness analysis}

We assessed costs from the hospital perspective using staffing costs from national pay scales and inpatient costs by hospital coding (spell-based NHS reference costs for 2011-12). Each spell was assigned a unit cost on the basis of its health resource group, and a total patient cost was calculated as the sum of costs across all spells. We adjusted costs for the differential input price (staff etc) in London and Brighton, using the Market Forces Factor. To match spells to reference costs, data for 2011-12 and 2012-13 were merged with the relevant year's reference costs using the hospital record health resource group code based upon admission method. Where no match was found for a 2012-13 spell, the data were merged into the 2011-12 reference costs (ie we assumed that the cost was unchanged). No discounting for time was invoked. Non-parametric bootstrapping was used to account for uncertainty in calculating incremental costeffectiveness ratios, using 1000 replications.

\section{Results}

Fig 1 shows the flow of participants. 1009 patients were referred, 387 of whom were ineligible. Of the 622 eligible patients, $66 \%$ agreed to participate. 414 patients provided consent, but three had already been randomly assigned and one was untraceable, leaving 410 for analysis. Table 1 shows the population characteristics. Most (81\%) were male and British (71\%). 178 (43\%) had been living on the street immediately before admission and most $(\mathrm{n}=331)$ had been previously admitted to hospital. Mental health disorders (74\% reported depression), infection ( $\sim 40 \%)$ and alcohol abuse (>30\%) were common; illicit drug use was admitted in a minority (Supplementary Table 1, S3).

Fig 2 illustrates the total number of days in hospital during the initial admission and following 90 days. 204 patients allocated to standard care had 324 admissions at an average duration of 8.8 days per admission, whereas 206 patients allocated to 


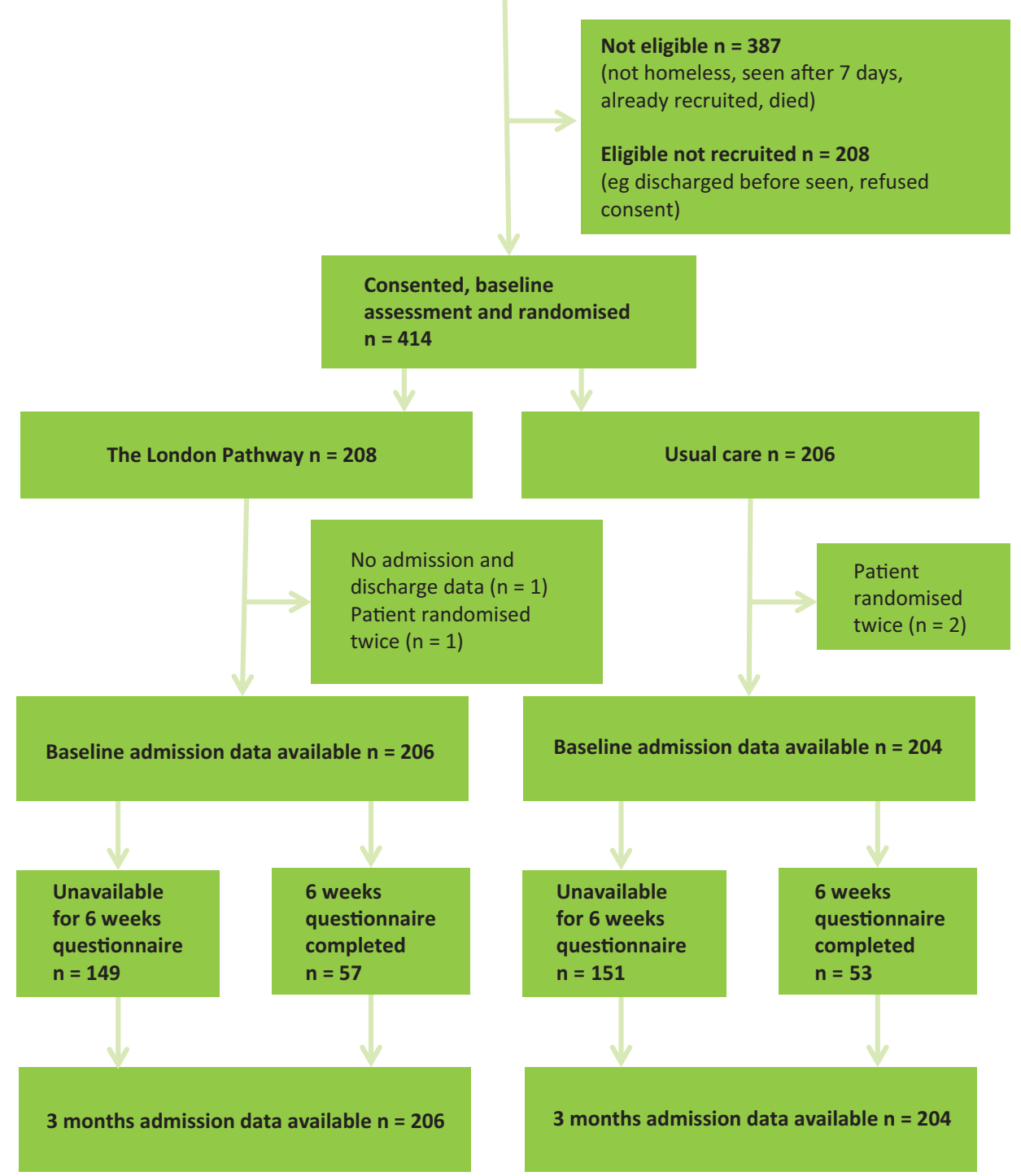

Fig 1. Trial profile.

enhanced care had 324 admissions and stayed an average of 8.5 days. The total length of time spent in hospital in 90 days was 14.0 and 13.3 days, respectively (adjusted difference -0.4 [ $95 \%$ CI -3.88 to 3.07$]$ ). Four patients in the enhanced care arm and two in the standard care arm were admitted for more than 90 days. Readmissions and reattendances at the emergency department did not differ between the groups (Table 2), and neither did planned as opposed to emergency readmissions (Supplementary Table 2, S3). Ten deaths occurred - seven with enhanced care and three with standard care arm. Following a change to the protocol after 6 months, patients were asked to consent to providing long-term follow-up data. 226 patients agreed to long-term follow-up and Table 2 shows no significant difference in the number of readmissions in the first year or in the total length of stay (20.8 days in the standard care arm vs 18.5 days in the enhanced care arm). The number of attendances to accident and emergency did not differ between the groups. There was a numerical reduction in the total number of attendances (bootstrapped) between the control and intervention groups (means of six and five attendances respectively), but it was not statistically significant (difference -0.8 [95\% CI -4.3 to 2.8$]$ ).

The impact of the intervention on quality of life and housing was assessed in $48(23.3 \%)$ patients allocated to standard care and $53(25 \%)$ in the enhanced care group. There were no differences in patients who did or did not complete the questionnaire (Supplementary Table 3, S3) and there was no relationship between follow-up duration and either the follow-up EQ-5D-5L index or the gain in EQ-5D-5L scores (Supplementary Fig 1, S3), suggesting that the benefits of the intervention did not decline with time. Table 3 compares EQ-5D-5L scores from admission and post-discharge questionnaires. There was an increase in EQ-5D-5L scores in patients who had received the intervention compared with 


\section{Table 1. Patient demographics.}

Male sex

Nationality

UK

European Union

Other/not given

\section{Asylum seeker - yes \\ Refugee - yes

$$
\begin{aligned}
& <1 \text { month } \\
& 1-12 \text { months } \\
& 1-5 \text { years } \\
& >5 \text { years }
\end{aligned}
$$$$
\text { Not given }
$$

Time since permanent accommodation

Long-term medical conditions - yes

Long-term mental health problems - yes

Housing status on admission

Street

Unstable address

Bed \& breakfast or hotel

Unsupported hostel

Supported hostel

Stable address*

Not given

\section{Hospital admission in previous 12 months}

None

1

2-9

10-30

Not given

\section{Nights sleeping rough in $\mathbf{2 8}$ days}

None

1-6 days

7-13 days

14 to 20 days

21 to 27 days

Every day

Not given

EQ-5D-5L Mean (SD)
Standard care $(n=204)$

$154(75.5 \%)$

$50(24.5 \%)$

$42.5(11.3)$

$166(81.4 \%)$

Enhanced care $(n=206)$

$156(75.7 \%)$

$50(24.3 \%)$

$41.6(12.1)$

$168(81.6 \%)$

$148(72.5 \%)$

$143(69.4 \%)$

$36(17.6 \%)$

$46(22.3 \%)$

$17(8.3 \%)$

$7(3.4 \%)$

$7(3.4 \%)$

$30(14.7 \%)$

$38(18.4 \%)$

$46(22.5 \%)$

$33(16.0 \%)$

$58(28.4 \%)$

$54(26.2 \%)$

$32(15.7 \%)$

$48(23.3 \%)$

$38(18.6 \%)$

$33(16.0 \%)$

$156(76.5 \%)$

$163(79.1 \%)$

$113(55.4 \%)$

$123(59.7 \%)$

$96(47.1 \%)$

$82(39.8 \%)$

$62(30.4 \%)$

$70(34.0 \%)$

$3(1.5 \%)$

$12(5.8 \%)$

$8(3.9 \%)$

$26(12.7 \%)$

$4(1.9 \%)$

$34(16.5 \%)$

$3(1.5 \%)$

$1(0.5 \%)$

$7(3.4 \%)$

$27(13.1 \%)$

$55(26.7 \%)$

$104(50.5 \%)$

$13(6.3 \%)$

$7(3.4 \%)$

$12(5.9 \%)$

$110(53.4 \%)$

$32(15.5 \%)$

$11(5.3 \%)$

$11(5.3 \%)$

$4(1.9 \%)$

$32(15.5 \%)$

$6(2.9 \%)$

$0.47(0.32)$

Data are $\mathrm{n}(\%)$ unless otherwise stated. *Includes patients who became homeless during admission. SD = standard deviation. 


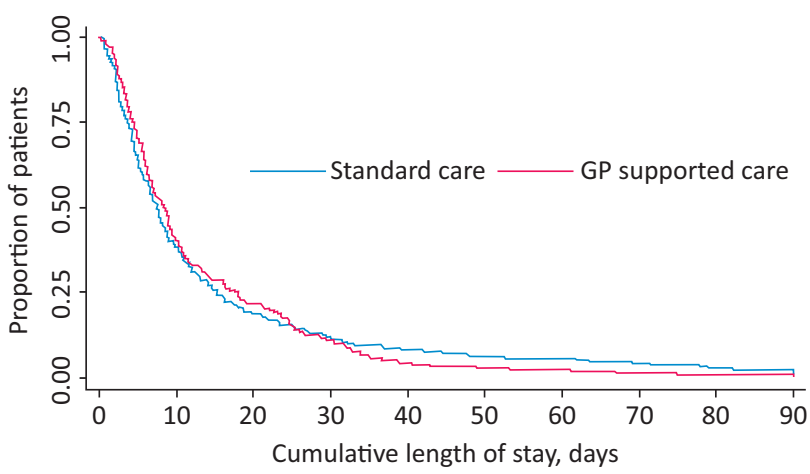

Fig 2. Kaplan-Meier curve showing cumulative length of admission within 90 days in the standard and enhanced care groups. those who received standard care, although this increase was not statistically significant. Table 3 also shows accommodation after discharge. $14.6 \%$ of patients in the control arm were street homeless at discharge compared with $3 \cdot 8 \%$ of patients in the intervention arm (odds ratio 0.14 [95\% CI 0.02-0.86], $\mathrm{p}=0.034$ ). To analyse the self-assessed sliding scale for coping, we used a bootstrapped linear regression model for the four coping questions (money, relationships, drugs and alcohol, and managing accomodation (supplementary questionaires, S1 and S2)) to compare follow-up score, adjusted for age, sex and site, and baseline score (Table 4). The intervention significantly improved scores for money and relationships and numerically improved the scores for accommodation, but did not affect the scores for drugs and alcohol. Taken together, these data indicate that the intervention improved accommodation and quality of life after discharge.

Table 2. Duration of in-patient stay, reattendances and A\&E attendances within 90 days and 1 year of admission. The total duration of in-hospital stay (the index admission plus readmissions) is shown for all patients by treatment arm. Type of admission, number of admissions and attendances in A\&E are also shown.

\section{Standard care $(n=204)$}

Part A: Within 90 days of admission (all patients)

Total admissions

Type of admissions

Emergency

Elective

Other/not known

Number of admissions within 90 days of index admission

1

$2-5$

$>6$

Mean total length of stay censored at 90 days (SD)

Patients attending A\&E within 90 days of discharge, $n$ ( \% )

Part B: Within 1 year of admission (additional consent obtained)

Total participants who consented to longer follow-up

Total admissions

Type of admissions

Emergency

Elective

Other/not known

Admissions in 1 year from index admission

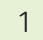

2-5

$>6$

Mean length of individual stay, days (SD)

Bootstrapped total length of stay, days (SE)

Patients attending A\&E within 1 year of consent, $n$ (\%)

Bootstrapped total attendances, $n$ (SE)
266

24

34

139

63

2

$14.0(18.5)$

$57(28 \%)$

Data are $n$ unless otherwise specified. $A \& E=$ accident and emergency; SD = standard deviation; $S E=$ standard error. 
Nigel Hewett, Peter Buchman, Jeflyn Musariri et al

Table 3. Impact of the intervention on quality of life and accommodation.

\begin{tabular}{|c|c|c|c|c|c|c|}
\hline & \multicolumn{2}{|c|}{ Standard care } & \multicolumn{2}{|c|}{ Enhanced care } & \multirow{2}{*}{$\begin{array}{l}\text { Adjusted difference at } \\
\text { follow-up ( } 95 \% \mathrm{CI})\end{array}$} & \multirow[t]{2}{*}{$\mathrm{p}$} \\
\hline & Baseline & Follow-up & Baseline & Follow-up & & \\
\hline \multicolumn{7}{|l|}{ EQ-5D-5L questionnaire } \\
\hline Patients, $\mathrm{n}$ & 48 & 48 & 53 & 53 & & \\
\hline Mean total EQ-5D-5L score & 0.48 & 0.47 & 0.43 & 0.56 & $0.09(-0.03$ to 0.22$)$ & 0.151 \\
\hline \multicolumn{7}{|l|}{$\begin{array}{l}\text { Accommodation } \\
\text { questionnaire }\end{array}$} \\
\hline Street homeless, n/N ( \% ) & $\begin{array}{l}96 / 204 \\
(47.1 \%)\end{array}$ & $\begin{array}{l}7 / 48 \\
(14.6 \%)\end{array}$ & $\begin{array}{l}82 / 206 \\
(39.8 \%)\end{array}$ & $2 / 53(3.8 \%)$ & $0.14(0.02$ to 0.86$)$ & 0.034 \\
\hline
\end{tabular}

\section{Cost-effectiveness}

The costs of the intervention were viewed from the hospital perspective. Staff costs included one full-time nurse and 16 GP hours per week. Multidisciplinary meeting costs were included in the hospital staff time allocation and external costs were excluded. We estimate that the intervention costs $£ 154,228$ to operate per year with the salary costs (Supplementary Table 5, S3) added to the training costs $(\mathfrak{1} 11,120)$. The impact of the intervention on inpatient activity was assessed by comparing average cost per inpatient spell using all spells for which data were available. We found no impact on inpatient costs and compared operating costs of the intervention with the effect on patient health gain, measured in quality-adjusted lifeyears (QALYs). At baseline, health-related quality of life was similar across the arms. Since there was no correlation between EQ-5D-5L change and duration of follow-up (Supplementary Table 5, S3), we translated the EQ-5D-5L index scores into QALYs by assuming that the patient stays at their baseline health state for the shortest follow-up duration in our sample (6 days), and the patient is assumed to move to their follow-up health state for a duration of the difference between the longest and shortest follow-up (235-6 = 229 days). This probably underestimates the intervention's health gain, because QALYs are censored at 235 days but the benefits could last longer. On the basis of these assumptions, we estimate an incremental costeffectiveness ratio of $£ 26,431$. Supplementary Fig 2 summarises the uncertainty around our estimate in a cost-effectiveness acceptability curve.

\section{Discussion}

This is the first randomised controlled trial of a hospitalbased, GP-led intervention in a homeless population. We found no significant difference in the duration of hospital stay between patients receiving enhanced care and those receiving standard care, refuting our hypothesis that the intervention would reduce length of stay. Long-term follow-up of a subset of patients showed no significant reduction in hospital service usage in those who received enhanced care. Both hospitals involved in this study provide acute medical care to large populations and both have been subjected to pressures to reduce length of stay and reduce admissions. It is therefore possible that our failure to reduce length of stay in homeless people reflects the severity of their disease on admission and the relative efficiency of the existing discharge service. On a number of different measures, the intervention improved quality of life, and this improvement was cost-effective using conservative assumptions. The benefits to patients exposed to the intervention were substantial, and this is one of the first interventions to show a reduction in the proportion of patients sleeping on the streets.

A strength of our trial was that it was a randomised, realworld, controlled clinical trial in a population who are not normally studied and in whom robust data collection is rare. However, the rate of recruitment was low, and our study in the self-selected population who participated should be interpreted with caution. We did not provide support at weekends or over holidays, which could have reduced the opportunity for a benefit. However, a 7-day service might prove unduly expensive. Although the study did not recruit as planned and was underpowered, the similar duration of hospital stay in 400 patients suggests that full recruitment to the study would not have altered our conclusions. The study involved an in-hospital study team interacting with the ward clinical staff, and the team could have increased awareness of the needs of homeless people and led to an improvement in care in the control population.

Table 4. Impact of the intervention on self-assessed sliding scale for coping with money, relationships, drugs and alcohol and accommodation. The sliding scale coping questionnaire is a 10-point questionnaire in which $1=$ not coping and $10=$ coping well. SD = standard deviation.

$\begin{array}{llllll} & \mathbf{n} & \text { Mean score (SD) } & \text { Mean score (SD) } & \text { Adjusted difference at follow-up } & \mathbf{p} \\ \text { Money } & 109 & 3.85(3.03) & 5.21(2.68) & 1.23(0.17 \text { to } 2.29) & 0.023 \\ \text { Relationships } & 108 & 4.79(3.36) & 5.68(2.97) & 1.23(0.10 \text { to } 2.36) & 0.032 \\ \text { Drugs and alcohol } & 106 & 7.45(3.10) & 7.33(2.94) & -0.03(-1.04 \text { to } 0.99) & 0.96 \\ \text { Accommodation } & 108 & 4.98(3.15) & 6.20(3.15) & 1.17(-0.06 \text { to 2.40) } & 0.062\end{array}$


It is challenging to maintain contact with patients. We used research fellows to contact patients, but fewer than 30\% responded, reducing the power of the QALY assessments. The patients we contacted were not atypical, and because patients from both trial arms were contacted with equal frequency we believe that the quality-of-life findings are valid. Analysis of quality-of-life data typically assumes that benefits accrue during the observation period. In our study, the EQ-5D-5L scores did not vary by duration of follow-up and we therefore assumed that the benefits accrued during admission persisted until the duration of the longest period of follow-up. This could reduce the benefits of the intervention as the observed improvements in accommodation are likely to last. Our data on QALY benefits from the intervention are rigorous, but likely to underestimate the gains.

\section{Interpretation}

This randomised controlled trial in patients who are homeless indicates that rigorous assessment in difficult-to-reach populations is feasible, albeit challenging. We evaluated a complex intervention and assessed the impact on length of stay, quality of life and costs. Previous studies have suggested that a homeless intervention team could reduce overall length of stay, but hospitals considering the Pathway approach have expressed concerns about a negative impact. Neither assumption is correct - the homeless intervention team neither increased nor decreased the duration of stay. Community interventions to improve care for homeless people and reduce their impact on the health service have been successful. For example, a Bradford study using hospital in-reach, an 'intermediate' care city centre bed facility, and a 'street medicine' approach showed a reduction in contact with emergency services. These studies suggest that community-based interventions could improve the care of homeless populations (Ashman, Smith, Goldman - data presented at the RCGP conference, Harrogate, 2015).

Despite the failure to demonstrate a reduction in hospital stay in our intervention, it is important to note the impact on quality of life and housing status. Previous studies ${ }^{14}$ have shown that a variety of interventions do not lead to improvements in housing status. Our data indicate that, for patients receiving the Pathway intervention, the proportion of people who return to the streets is reduced. However, the number of street homeless patients was relatively few and further studies will be required to confirm this important benefit.

\section{Funding}

The study was funded by the National Institute for Health Research.

\section{Acknowledgements}

The views expressed are those of the authors and do not necessarily represent the views of the NIHR.

\section{Supplementary material}

Additional supplementary material may be found in the online version of this article at www.clinmed.rcpjournal.org:

S1 - Admission questionnaire

S2 - Follow-up questionnaire

S3 - Supplementary tables and figures

\section{References}

1 Morrison DS. Homelessness as an independent risk factor for mortality: results from a retrospective cohort study. Int J Epidemiol 2009;38:877-83.

2 Hwang SW, Wilkins R, Tjepkema M, O'Campo PJ, Dunn JR. Mortality among residents of shelters, rooming houses, and hotels in Canada: 11 year follow-up study. BMJ 2009;339:b4036.

3 Hwang SW, Martin RE, Tolomiczenko GS, Hulchanski JD. The relationship between housing conditions and health status of rooming house residents in Toronto. Can J Public Health 2003;94:436-40.

4 Chambers C, Chiu S, Katic M et al. High utilizers of emergency health services in a population-based cohort of homeless adults. Am J Pub Health 2013;103(suppl 2):S302-10.

5 Department of Health. Healthcare for single homeless people. London: Department of Health, 2010.

6 Hewett NC. How to provide for the primary health care needs of homeless people: what do homeless people in Leicester think? $\mathrm{Br} J$ Gen Pract 1999;49:819.

7 McHugo GJ, Bebout RR, Harris M et al. A randomized controlled trial of integrated versus parallel housing services for homeless adults with severe mental illness. Schizophrenia Bull 2004;30:969-82.

8 Somers JM, Moniruzzaman A, Palepu A. Changes in daily substance use among people experiencing homelessness and mental illness: 24 -month outcomes following randomization to housing first or usual care. Addiction 2015;110:1605-14.

9 Somers JM, Patterson ML, Moniruzzaman A et al. Vancouver At Home: pragmatic randomized trials investigating Housing First for homeless and mentally ill adults. Trials 2013;14:365.

10 Wolitski RJ, Kidder DP, Pals SL et al. Randomized trial of the effects of housing assistance on the health and risk behaviors of homeless and unstably housed people living with HIV. AIDS Behav 2010;14:493-503.

11 Hwang SW, Burns T. Health interventions for people who are homeless. Lancet 2014;384:1541-7.

12 Hewett N, Halligan A, Boyce T. A general practitioner and nurse led approach to improving hospital care for homeless people. BMJ 2012;345:e5999.

13 Herdman M, Gudex C, Lloyd A, et al. Development and preliminary testing of the new five-level version of EQ-5D (EQ-5D-5L). Qual Life Res 2011;20:1727-36.

14 Meschede T. Accessing housing:exploring the impact of medical and substance abuse services on housing attainment for chronically homeless street dwellers. Behav Soc Environ 2010;20:153-69.

Address for correspondence: Prof GR Foster, Queen Mary University of London, Blizard Institute, 4 Newark Street, London E1 4AT, UK.

Email: g.r.foster@qmul.ac.uk 